\title{
Fluorescence Study on the Dynamics and Structure of Polymer Chain I. Intramacromolecular Photo-crosslinking Reaction of a Polymer Containing Anthryl and Eosinyl Moieties in Dilute Solution
}

\author{
Toshinori TorII, ${ }^{\dagger}$ Hideharu UsHIKI, and Kazuyuki HORIE* \\ Faculty of General Education, Tokyo University of Agriculture and Technology, 3-5-8, \\ Saiwai-cho, Fuchu-shi, Tokyo 183, Japan \\ * Faculty of Engineering, University of Tokyo, 7-3-1, Hongo, \\ Bunkyo-ku, Tokyo 113, Japan
}

(Received March 4, 1992)

\begin{abstract}
A photo-crosslinkable polymer with pendant anthryl and eosinyl moieties attached to its side chains was synthesized. Dilute DMF ( $N, N$-dimethyl formamide) solutions of the polymer were UV-irradiated to induce intramacromolecular photo-crosslinking reaction under degassed conditions. Decrease in anthryl moiety based on photo-dimerization between anthryl moieties during the UV-irradiation was evaluated from the change in absorption spectra. Change in the dimensions of the polymer coil induced by the reaction was studied by GPC. Parameters for polymer chain dynamics were studied in the course of the intramacromolecular crosslinking reaction resulting in shrinkage of the polymer coil. The time-conversion curve of the photodimerization between anthryl moieties showed that the photo-crosslinking reaction consists of two stages. The mobility of polymer chain segments decreased gradually in the initial stage and deceases rapidly in the later stage. Radius of gyration of the polymer coil and the rotational mobility of side chain decreased rapidly only in the later stage. Three possible models for the reaction are discussed in order to explain the results. The short range reaction model is most suitable model for the intramacromolecular crosslinking reaction. In the initial stage crosslinks are formed between nearest-neighbor side chains; in the later stage shrinkage of the polymer coil occurs due to formation of crosslinks between far-apart side chains which remained unreacted in the initial stage.
\end{abstract}

KEY WORDS Photo-crosslinkable Polymer / Intramolecular Photo-crosslinking / Emission Probe Method / Polymer Chain Dynamics / Dimension of Polymer /

Photo-crosslinking reactions in polymer systems with various chromophores have been reported by many authors. Reiser et $a .^{1-4}$ studied photocycloaddition of poly(vinyl cinnamate) $(\mathrm{PVCm})$ in amorphous solids. In solid PVCm, photo-crosslinking are produced by intermolecular reaction as a result of the high degree of polymer interpenetration. It has been shown that the quantum yield for the photocycloaddition decreases with the extent of reaction so that only about half the cinnamoyl moieties are capable of undergoing the photocycloaddition. They explained this phenomenon from the view points of reaction sites with the distributed reactivity in the solid state.

Sindo et al. $^{5-7}$ studied intra- and intermolecular photo-crosslinking reactions in solution of polymers containing cinnamoyl moieties. They reported that the quantum yield for the intramolecular crosslinking increased and microgel abundant in intramolec-

\footnotetext{
† Present address: Faculty of Engineering, University of Tokyo, 7-3-1, Hongo, Bunkyo-ku, Tokyo 113, Japan.
} 
ular crosslinks were produced with increase in molecular weight, density of reactive side chain, and decrease in concentration of the polymer. In such studies on the photocrosslinking reaction of polymers, however, only applications to technology have been emphasized. No attention has been paid to the relationship between polymer chain dynamics and crosslinking reaction or crosslinks in a polymer during such the reactions.

The emission probe method has been developed in the field of polymer science. ${ }^{8}$ The emission characteristics of probes are useful to reflect microenviornments in polymer systems. The emission probe method provides answers to questions of flexibility or dynamics, free volume, microviscosity, conformation, and physical characteristics in polymer systems. An emission probe was used here for characterizing the polymer chain dynamics during structure change due to a intramacromolecular photo-crosslinking reaction.

\section{EXPERIMENTAL}

\section{Materials}

The Photo-crosslinkable Polymer. Styrene and chloromethyl styrene were copolymerized in benzene with azobis(isobutyro-nitrile) as an initiator at $70^{\circ} \mathrm{C} .{ }^{9}$ The precipitated polymer, copoly(St/CMSt), was reprecipitated three times from benzene into methanol, and then dried at room temperature.

To label the photo-crosslinkable polymer with the emission probe, $1.0 \mathrm{~g}$ of the copoly(St/CMSt) was dissolved to $10 \mathrm{ml}$ of DMF then $0.8 \mathrm{~g}$ of $2^{\prime}, 4^{\prime}, 5,7^{\prime}$-tetrabromofluorescein disodium salt (eosin $\mathrm{Y}$ ) and $0.75 \mathrm{~g}$ of sodium 9 -anthracenecarboxylate ${ }^{10}$ were added to the solution. The solution was stirred for $6 \mathrm{~h}$ at room temperature in the dark. It was poured into methanol, and the precipitate was filtered, washed with methanol, and dried under vacuum.

The number average molecular weight was $2.9 \times 10^{6}$. Numbers of two pendant moieties in a polymer chain were determined from the absorbances of chromophores in DMF solution at $365 \mathrm{~nm}$ (anthryl) and $510 \mathrm{~nm}$ (eosinyl) on the basis of molar extinction coefficients of the corresponding model compounds. The numbers of anthryl and eosinyl moieties in a polymer chain were $1.3 \times 10^{3}$ $(5 \%)$ and $1.8 \times 10^{2}(0.7 \%)$, respectively.

Model Compounds. Benzyl 9-anthracenecarboxylate, a model compound for the photocrosslinkable side chain, was prepared by esterification of sodium 9-anthracenecarboxylate with benzyl chloride in DMF solution. $2^{\prime}, 4^{\prime}, 5^{\prime}, 7^{\prime}$-tetrabromofluorescein benzylester sodium salt (benzyl eosinate), a model compound having a structure very similar to the emission probe side chain, was prepared from eosin $\mathrm{Y}$ and benzyl chloride by the similar esterification procedure. The characterization of two model compounds was performed by IR spectroscopy. Absorption bands at $1540 \mathrm{~cm}^{-1}$ attributed to carboxylate ion of sodium 9-anthracenecarboxylate disappeared and four absorption bands attributed to aromatic ester appeared for the model compound to the anthryl side chain. Similar behavior was observed in preparation of the model compound to the eosinyl side chain. Molar extinction coefficients of the two chromophores were determined to be $5.3 \times$ $10^{3} \mathrm{dm}^{3} \mathrm{~mol}^{-1} \mathrm{~cm}^{-1}$ at $365 \mathrm{~nm}$ for benzyl 9-anthracenecarboxylate and $3.0 \times 10^{4} \mathrm{dm}^{3}$ $\mathrm{mol}^{-1} \mathrm{~cm}^{-1}$ at $510 \mathrm{~nm}$ for benzyl eosinate, respectively. Figure 1 shows the polymer and model compounds.

DMF solutions of the photo-crosslinkable polymer $\left(0.4 \mathrm{~g} \mathrm{dm}^{-3}\right)$ in pyrex cells were degassed by several freeze-pump-thaw cycles and sealed under high vacuum. The solution containing benzyl 9-anthracenecarboxylate $\left(2.0 \times 10^{-4} \mathrm{~mol} \mathrm{dm}^{-3}\right)$ and benzyl eosinate $\left(1.0 \times 10^{-5} \mathrm{~mol} \mathrm{dm}^{-3}\right)$ in a pyrex cell was degassed and sealed off under the same conditions. 

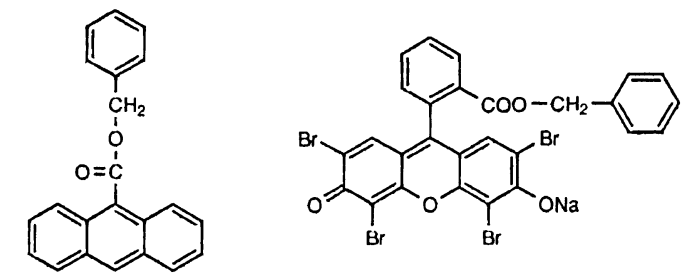

benzyl anthracenecarboxylate benzyl eosinate

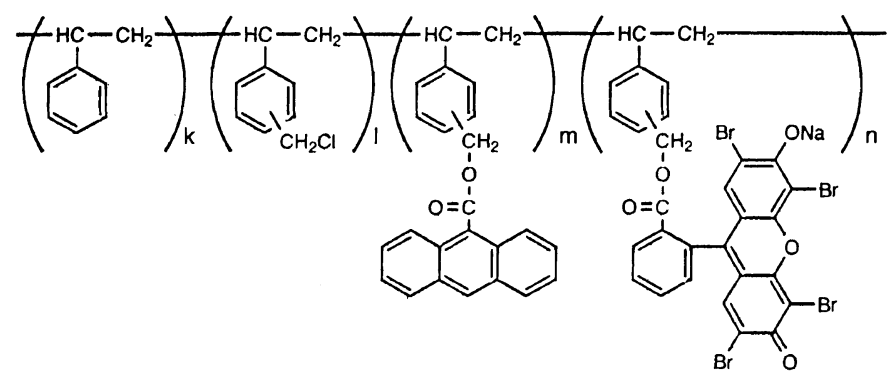

photo-crosslinkable polvmer

Figure 1. Chemical structures of model compounds and photo-crosslinkable polymer.

\section{Instruments}

Degassed polymer solutions were UVirradiated at room temperature by light from a super-high-pressure $\mathrm{Hg}$ lamp (Toshiba SHL-100UV). In order to induce photodimerization among anthryl moieties, light ranging from $320 \mathrm{~nm}$ to $420 \mathrm{~nm}$ was used for irradiation using two glass filters (Toshiba UV-35 and UV-D35). Absorption spectra were measured on a modified Hitachi U-1100 type spectrophotometer combined with a personal computer NEC PC-9801 through an RS-232C interface. Fluorescence spectra and fluorescence anisotropy ratio were measured with a Hitachi 650-40 type fluorescence spectrophoto-meter equipped with a Hitachi 650-0178 type data processor. Excited singlet lifetimes of two chromophores were measured with a Horiba NEAS-1100 type time-resolved fluorescence spectro-photometer. All spectroscopic measurements were carried out under degassed conditions. The GPC measurements were carried out with the computer assisted GPC system that was designed in our laboratory. All data obtained were analyzed by using a personal computer (NEC PC-9801) whose program softwares were prepared in our laboratory.

\section{RESULTS AND DISCUSSION}

\section{Model Compounds}

The change in absorption spectra of the DMF solution containing the model compounds under UV-irradiation $(320 \mathrm{~nm}$ to $420 \mathrm{~nm}$ ) is shown in Figure 2. The absorption band ranging from $300 \mathrm{~nm}$ to $400 \mathrm{~nm}$ is mainly ascribed to benzyl 9-anthracenecarboxylate (BA). Benzyl eosinate absorbs in the region from $450 \mathrm{~nm}$ to $570 \mathrm{~nm}$ and slightly also in the region from $300 \mathrm{~nm}$ to $400 \mathrm{~nm}$. Therefore, the concentration of BA was evaluated from corrected absorbance at $365 \mathrm{~nm}$. The change in the corrected absorbance of BA against UV-irradiation time, and second order plots for photo-dimerization of BA are shown in Figure 3, assuming that concentration of BA in the excited state is proportional to that of 


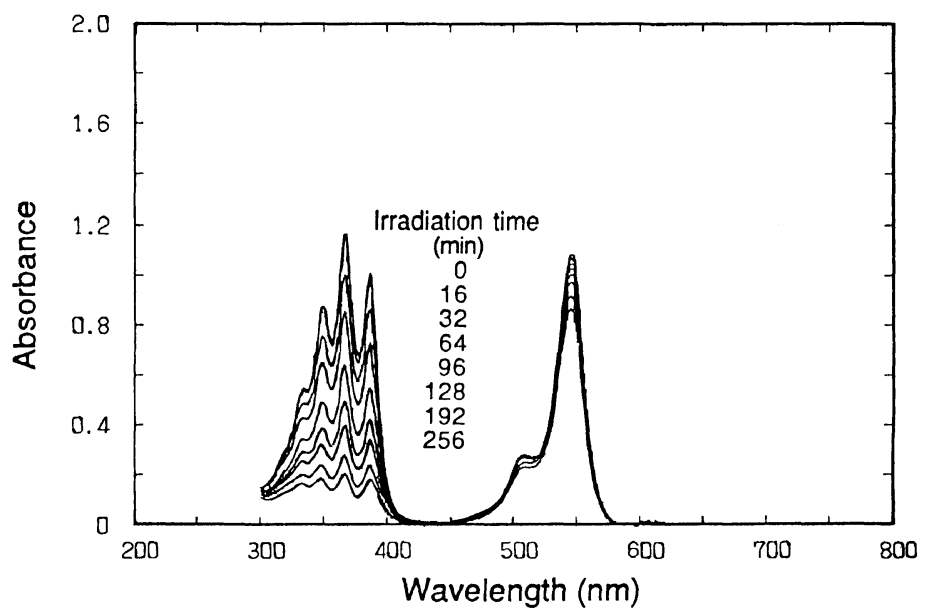

Figure 2. Change in absorption spectra of the DMF solution containing model compounds during UV-irradiation $(320 \mathrm{~nm}$ to $420 \mathrm{~nm})$. The initial concentrations of model compounds were $2.0 \times$ $10^{-4} \mathrm{~mol} \mathrm{dm}^{-3}$ for benzyl 9-anthracenecarboxylate and $1.0 \times 10^{-5} \mathrm{moldm}^{-3}$ for benzyl eosinate, respectively.

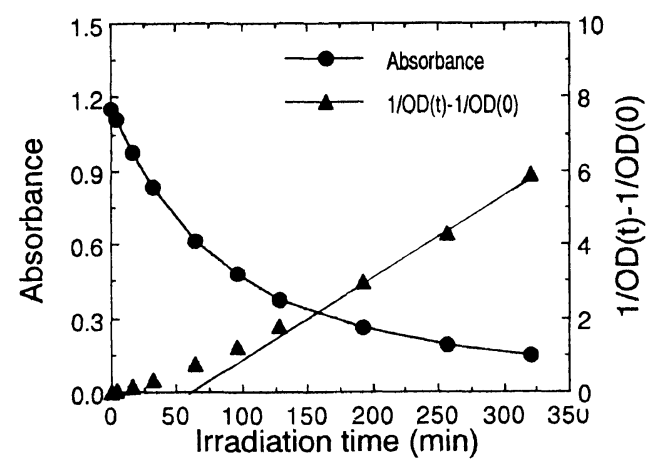

Figure 3. Photo-dimerization of benzyl 9-anthracenecarboxylate in DMF solution.

BA in the ground state. Photo-dimerization is a reaction between excited $\mathrm{BA}$ and $\mathrm{BA}$ in the ground state. When absorbance is large the concentration of excited BA is not proportional to the concentration of $\mathrm{BA}$ in the ground state. Thus apparent reaction rate tends to be small in the early stage of the reaction with large absorbance. The deviation from second-order kinetics is due to the large absorbance of the solution.

\section{The Photo-crosslinkable Polymer Solution}

The decrease in anthryl moiety based on photo-dimerization between anthryl side chains during the UV-irradiation was evaluated from change in absorption spectra. The change in absorption spectra of the polymer solution under UV-irradiation ( $320 \mathrm{~nm}$ to $420 \mathrm{~nm}$ ) is shown in Figure 4. The relationship between UV-irradiation time and corrected absorbance at $365 \mathrm{~nm}$ of anthryl moiety in the polymer and its conversion are shown in Figure 5. From GPC measurements and the analysis mentioned below, the initial radius of gyration of the polymer was determined to be $63 \mathrm{~nm}$. By using this value the critical overlap concentration, $c^{*}$, is estimated to be $19 \mathrm{~g} \mathrm{dm}^{-3}$. The concentration of the polymer was $0.4 \mathrm{~g} \mathrm{dm}^{-3}$ in the experimental system and much smaller than the value of $c^{*}$. Moreover no remarkable decrease in GPC elution volume during UV-irradiation was observed. Therefore the photo-crosslinking reaction proceeded only in individual polymer coils. We don't know so far the reason of decreasing eosinyl moiety in the later stage of the photo-crosslinking reaction.

The concentration of the anthryl moiety decreased so greatly that it is difficult to describe the reaction as a pseudo first-order reaction and the photo-crosslinking reaction 


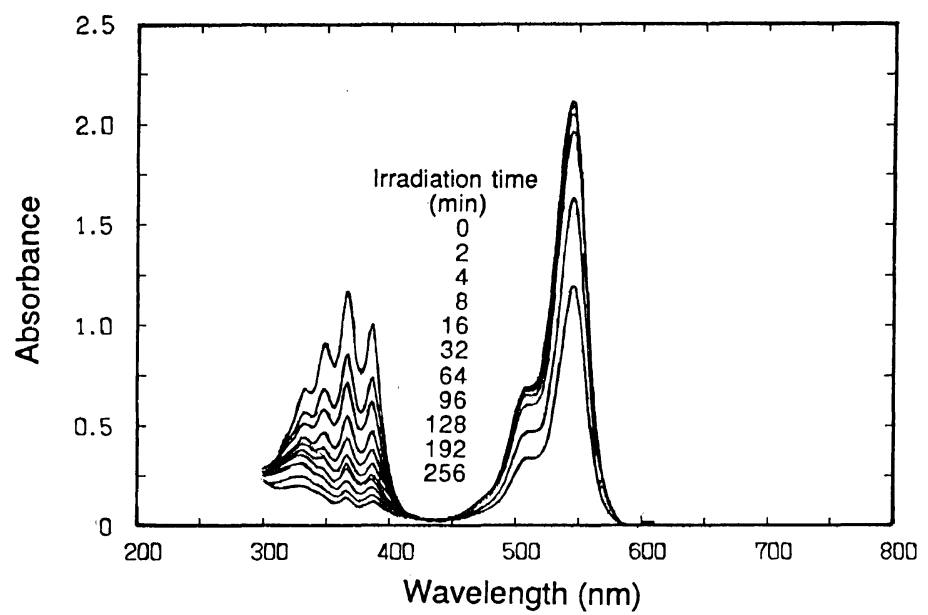

Figure 4. Change in absorption spectra of the DMF solution containing the photo-crosslinkable polymer solution during UV-irradiation $(320 \mathrm{~nm}$ to $420 \mathrm{~nm})$. The concentration of the polymer was $0.4 \mathrm{~g} \mathrm{dm}^{-3}$.

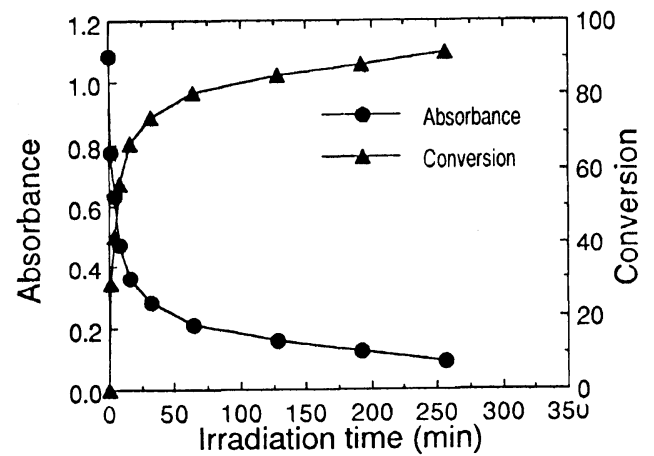

Figure 5. Changes in corrected absorbance at $365 \mathrm{~nm}$ and the conversion of anthryl moiety in the polymer during UV-irradiation.

should be considered to take place between two anthryl moieties in side chain of the same polymer coil. Both first-order and second-order plots of the reaction are shown in Figure 6. Both curves can be fitted by two lines, which denotes that the photo-crosslinking reaction consists of two stages. Because of the existence of two stages in the reaction, values for polymer chain dynamics should be discussed in terms of extent of the reaction rather than UV-irradiation time.

Change in dimension of polymer induced by

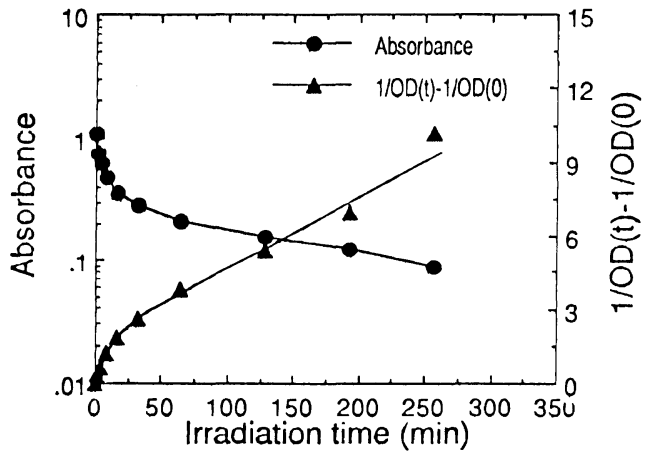

Figure 6. First and second-order plots for the photocrosslinking reaction of anthryl moiety in the polymer.

the intramacromolecular photo-crosslinking reaction was monitored with GPC. Figure 7 shows the relationship between average elution volume obtained from the computer-assisted GPC and conversion of anthryl moiety in the polymer. The average elution volume increased only in the later stage showing that only intramacromolecular photo-crosslinking reaction took place and that the polymer coils shrank only in the later stage.

In order to obtain parameters for polymer chain dynamics, fluorescence anisotropy ratio and excited singlet lifetime for each chro- 


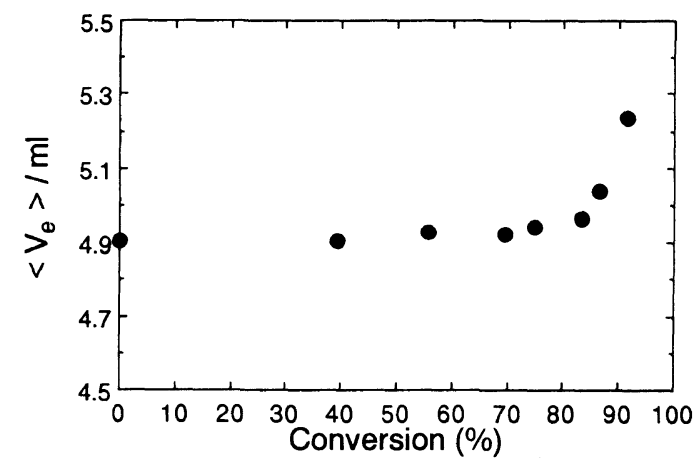

Figure 7. Change in average GPC elution volume, $\left\langle V_{\mathrm{e}}\right\rangle$, in accordance with the intramacromolecular photocrosslinking reaction.

mophore were measured. The fluorescence anisotropy ratio, $r$, was measured and calculated from four emission spectra using eq 1.

$$
r=\left(I_{\mathrm{VV}}-G I_{\mathrm{VH}}\right) /\left(I_{\mathrm{VV}}+2 G I_{\mathrm{VH}}\right) \quad G=I_{\mathrm{HV}} / I_{\mathrm{HH}}
$$

In this equation subscripts represent orientations of polarizers ( $V$ is vertical and $H$ is horizontal, respectively), located in front of (the first subscript) and behind (the second subscript) of the cell. The relationship between the conversion of anthryl moiety in the polymer and fluorescence anisotropy ratio, $r$, of each chromophore is shown in Figure 8. The value of $r$ for the eosinyl moiety changed in the later stage, but the value for the anthryl moiety changed slightly. All decay curves of fluorescence for both fluorophores were fitted single exponential functions. In Figure 9, excited singlet lifetime, $\tau_{\mathrm{s}}$, of each chromophore is plotted against the conversion of anthryl moiety in the polymer. Excited singlet lifetime of eosinyl moiety did not change remarkably. On the other hand, the lifetime of the anthryl moiety became longer in the later stage.

\section{Analysis}

Dimensions of the Polymer Coil. The shrinkage of the polymer coil was induced by the intramacromolecular photo-crosslinking reac-

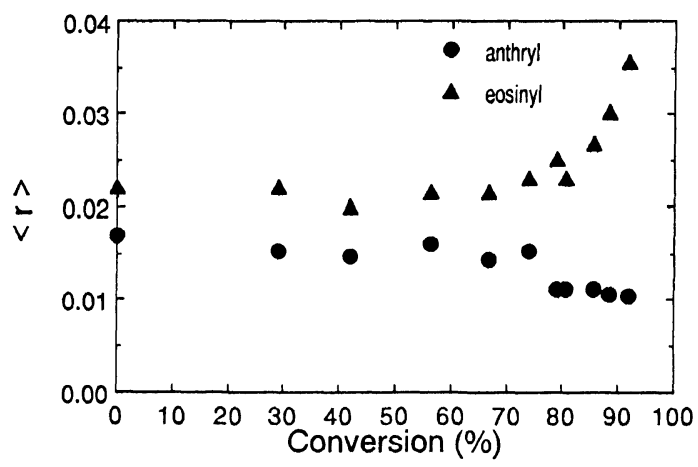

Figure 8. Plots of fluorescence anisotropy ratio, $r$, for anthryl (O) and eosinyl (A) moiety against the conversion of anthryl moiety.

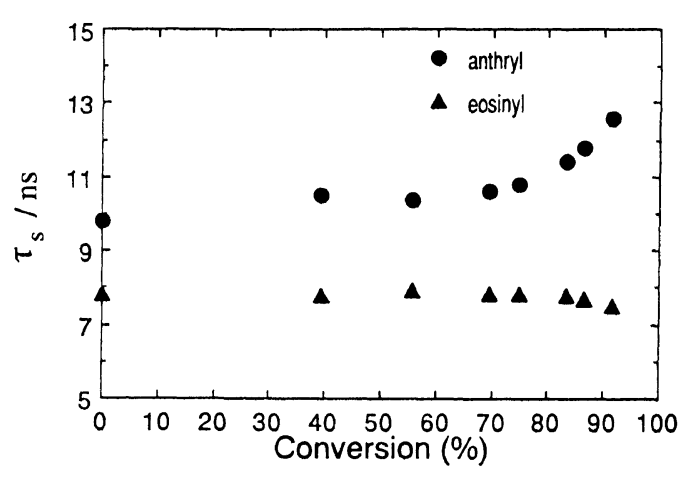

Figure 9. Plots of excited singlet lifetimes, $\tau_{\mathbf{s}}$, for anthryl (O) and eosinyl (A) moiety against the conversion of anthryl moiety.

tion. In order to know the change in mean radius of gyration of the polymer coil the GPC data were analyzed and the mean radius of gyration was used for estimating parameters for polymer chain dynamics.

For a given GPC column, the relationship between GPC elution volume, $V_{\mathrm{e}}$, and the product of intrinsic viscosity and molecular weight of a polymer, $[\eta] M$, is known to be universal for all types of polymers (e.g., linear, comb, star, and ladder polymers). ${ }^{11}$ Then we can use the GPC as a size exclusion chromatography. At first GPC measurements for poly(styrene) standards were carried out to obtain the universal calibration curves between $V_{\mathrm{e}}$ and $[\eta] M$ for the column used. The 
relationship between intrinsic viscosity and molecular weight of polystyrene in $\mathrm{DMF}$ solution at $40^{\circ} \mathrm{C} \quad\left([\eta]=2.29 \times 10^{-4} \mathrm{M}^{0.625}\right)$ was used. Then by using calibration curve, we transformed the value of $V_{\mathrm{e}}$ for the photocrosslinkable polymer measured with GPC into the $[\eta] M$.

The Flory-Fox's equation ${ }^{12}$ gives

$$
[\eta] M=\Phi_{\mathrm{F}}\left\langle r^{2}\right\rangle^{3 / 2}=6^{3 / 2} \Phi_{\mathrm{F}}\left\langle S^{2}\right\rangle^{3 / 2}
$$

where $\left\langle r^{2}\right\rangle$ is mean square end-to-end distance and $\left\langle S^{2}\right\rangle^{1 / 2}$ is mean radius of gyration of a polymer coil. For non-draining polymer coils, $\Phi_{\mathrm{F}}$ is constant to all kind of polymers regardless of their structures. Thus $\left\langle S^{2}\right\rangle^{1 / 2}$ was obtained from the eq 2 .

Rotational Motion of the Eosinyl Side Chain. Rotational diffusion coefficients of eosinyl side chain during the reaction were evaluated from fluorescence anisotropy ratios in the photostationary state and by decay times of eosinyl moieties.

The depolarization of fluorescences is caused by three phenomena. The first is intramolecular relaxation in the excited state, the second is energy transfer among fluorophores (Foerster type energy transfer), the third is rotational Brownian motion of fluorophore in the excited state. For the eosinyl moieties attached to the polymer as emission probe the mean distance between them is estimated to be $13 \mathrm{~nm}$. Because the distance is longer than the Foerster radius for general singlet-singlet energy transfer, no energy transfer among eosinyl moieties is expectable. Therefore the relationship between fluorescence anisotropy ratio and the mean relaxation time of rotation, $\rho_{\mathrm{r}}$, of a chromophore is given by ${ }^{13}$

$$
r_{0} / r=1+\left(3 \tau_{\mathrm{s}} / \rho_{\mathrm{r}}\right)
$$

where $\tau_{\mathrm{s}}$ is excited singlet lifetime of a fluorophore and $r_{0}$ is fluorescence anisotropy ratio of the chromophore in a rigid solution free from rotational motion. The relationship between $\rho_{\mathrm{r}}$ and the rotational diffusion coefficient, $D_{\mathrm{r}}$, for a rigid spherical body can be described by

$$
D_{\mathrm{r}}=1 /\left(2 \rho_{\mathrm{r}}\right)
$$

The value of $r_{0}$ for eosinyl moieties in the polymer side chain was assumed to be the same as for eosin $Y$, and was evaluated by using the method reported by Ushiki et al. ${ }^{14}$ In a glycerol-water solution system, the value of $r_{0}$ for eosin $Y$ was 0.29 . The values of the rotational diffusion coefficients, $D_{\mathrm{r}}$, of eosinyl moiety in the course of the photo-crosslinking reaction were calculated using eq 3 and 4 .

Translational Motion of Chain Segments. The translational diffusion coefficient of chain segments was estimated based on an energy transfer from the anthryl to the eosinyl moieties using mean radius of gyration of the polymer coil.

The critical radius of energy migration among anthryl moieties was reported to be $4.8 \mathrm{~nm}$ for poly[(10-phenyl-9-anthryl)-methyl methacrylate $].{ }^{15}$ On the other hand, the mean distance between anthryl moieties in the sample polymer was calculated to be $9.1 \mathrm{~nm}$. This distance is much larger than the critical radius of the energy migration. Therefore the energy migration can be ignored. The fluorescence band of the anthryl moiety, however, overlaps on the absorption band of the eosinyl moiety and fluorescence of the eosinyl moiety was observed when excitation light was $360 \mathrm{~nm}$. The fact shows that energy of excited anthryl moieties transfers to eosinyl moieties. So we consider the Foerster type energy transfer from the anthryl moieties to the eosinyl moieties. According to the Sterm-Volmer equation, the excited singlet lifetime of a chromophore, $\tau_{\mathrm{s}}$, is related to the rate constant for energy transfer, $k_{\mathrm{tr}}$, and the concentration of the energy acceptor, [E], by

$$
1 / \tau_{\mathrm{S}}=1 / \tau_{\mathrm{S} 0}+k_{\mathrm{tr}}[\mathrm{E}]
$$

where $\tau_{\mathbf{s} 0}$ is the excited singlet lifetime of the chromophore in the absence of the energy acceptor.

There are two causes for elongation of 
excited state lifetime. The first is decrease in the local concentration of the energy acceptor in the sample polymer coil, and the second is decrease in the value of $k_{\mathrm{tr}}$ due to decrease in the possibility of approach to induce the energy transfer from the excited chromophore to the energy acceptor. As shown in Figure 10, the radius of gyration of the polymer coil decreases only in the later stage. In Figure 9 $\tau_{\mathrm{S}}$ of the anthryl moiety became longer though the local concentration of the eosinyl moiety as the energy acceptor in individual polymer coils increased due to the shrinkage of polymer coil. Hence, the elongation of $\tau_{\mathrm{S}}$ for the anthryl moiety is considered to be caused by the decrease in rate constant of the energy transfer, $k_{\mathrm{tr}}$.

Local concentration of the eosinyl moiety as an energy acceptor in individual polymer coils, [E], can be estimated from the values of $\left\langle S^{2}\right\rangle^{1 / 2}$ mentioned above and we obtain the $k_{\mathrm{tr}}$ by

$$
\begin{aligned}
k_{\mathrm{tr}}= & \left(1 / \tau_{\mathrm{S}}-1 / \tau_{\mathrm{S} 0}\right) /[\mathrm{E}] \\
& {[\mathrm{E}]=3 N /\left(4 \pi N_{\mathrm{A}}\left\langle S^{2}\right\rangle^{3 / 2}\right) }
\end{aligned}
$$

where $N$ is the number of eosinyl moieties in a polymer coil and $N_{\mathrm{A}}$ is the Avogadro's number. The value of $k_{\mathrm{tr}}$ was evaluated by using values of excited singlet lifetime of the anthryl moieties in the polymer and $14.5 \mathrm{~ns}$ was used for the value of $\tau_{\mathrm{so}}$.

Smoluchowski proposed that the rate constant in a diffusion-controlled bimolecular reaction, $k_{2}$, is a function of translational diffusion coefficient, $D$, and critical reaction radius, $R .^{16}$

$$
k_{2}=4 \pi R\left(D+D^{\prime}\right)
$$

Because the energy transfer from an anthryl moiety to an eosinyl moiety is a bimolecular reaction, by assuming that the translational motion of side chains is identical to that of the chain segments, we obtain $k_{\mathrm{tr}}$ as follows

$$
D_{\mathrm{S}}=k_{\mathrm{tr}} / 8 \pi R
$$
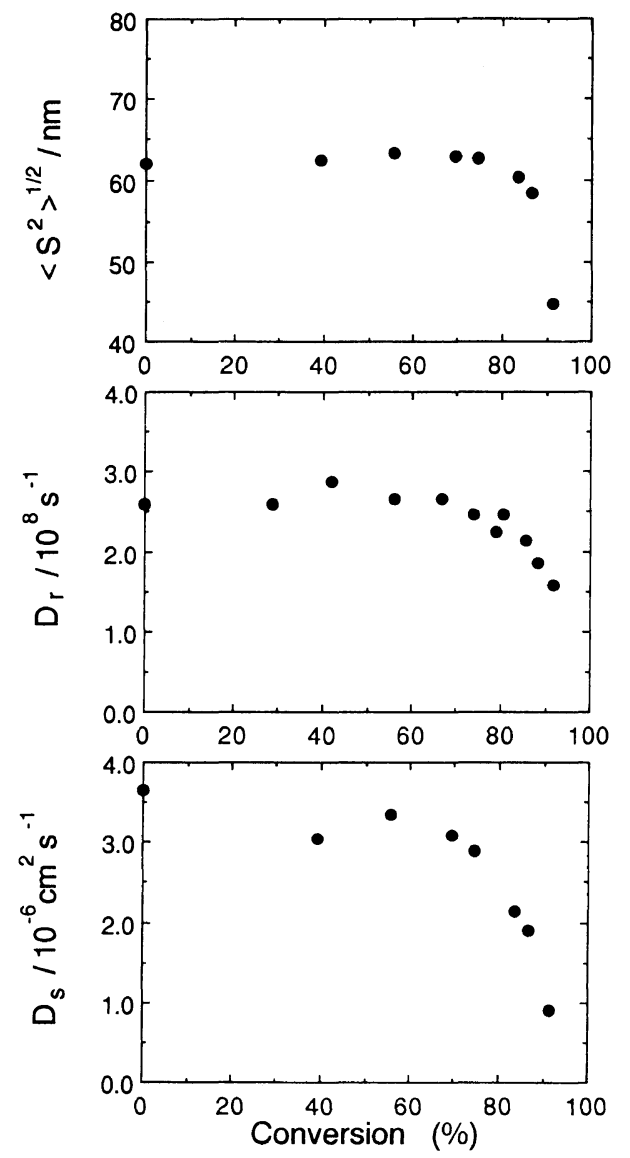

Figure 10. Changes in $\left\langle S^{2}\right\rangle^{1 / 2}, D_{\mathrm{r}}$, and $D_{\mathrm{s}}$ against the conversion of anthryl moieties in the polymer. $\left\langle S^{2}\right\rangle^{1 / 2}$, mean gyration radius of polymer coil; $D_{\mathrm{r}}$, rotational diffusion coefficient of eosinyl moiety; $D_{\mathrm{s}}$, translational diffusion coefficient of chain segments.

Then the translational diffusion coefficient of chain segments, $D_{\mathrm{S}}$, was estimated from equations 6 and 8 by assuming the value of $R$ as $2 \mathrm{~nm}$.

The relationships between conversion of anthryl moieties in the polymer and the values of $\left\langle S^{2}\right\rangle^{1 / 2}, D_{\mathrm{r}}$ and $D_{\mathrm{s}}$ are shown in Figure 10 . As shown in Figure 6, the intramacromolecular photo-crosslinking reaction consists of the fast and the slow reaction, in the initial and in the later stages respectively. The values of $D_{\mathrm{r}}$ and $\left\langle S^{2}\right\rangle^{1 / 2}$ slightly changed in the initial stage, and decreased rapidly in the later 
stage. The slow photo-crosslinking reaction in the later stage strongly affects the structure of the polymer coil and polymer chain dynamics. This rapid decrease in the values of $D_{\mathrm{r}}$ is attributed to the suppression of the rotational motion of side chains due to the shrinkage of the polymer coil in the later stage. The values of $D_{s}$, however, decrease gradually in the initial stage and decrease rapidly in the later stage. Translational motion of chain segments may be more sensitive to the formation of crosslinks.
Mechanism of the Intramacromolecular Photocrosslinking Reaction

The diffusion-controlled reaction of polymers in solution is distinguished from general chemical reaction because the process is greatly influenced by structure of a polymer, the polymer chain dynamics, viscosity of a solvent, and interaction to a solvent. ${ }^{16}$ Thus the reaction rate is a function of the structure of the polymer, position of reactive moieties on the polymer chain, and flexibility of the polymer chain. We propose the following three possible models for an intramolecular
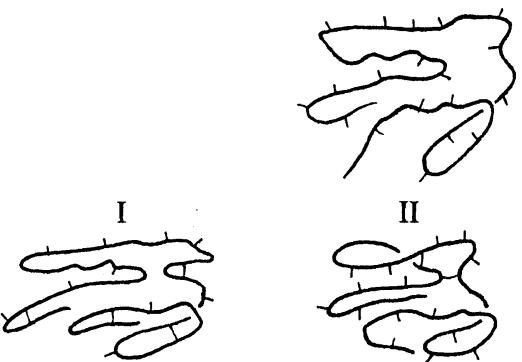

II

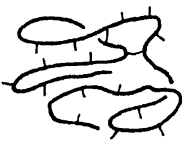

III
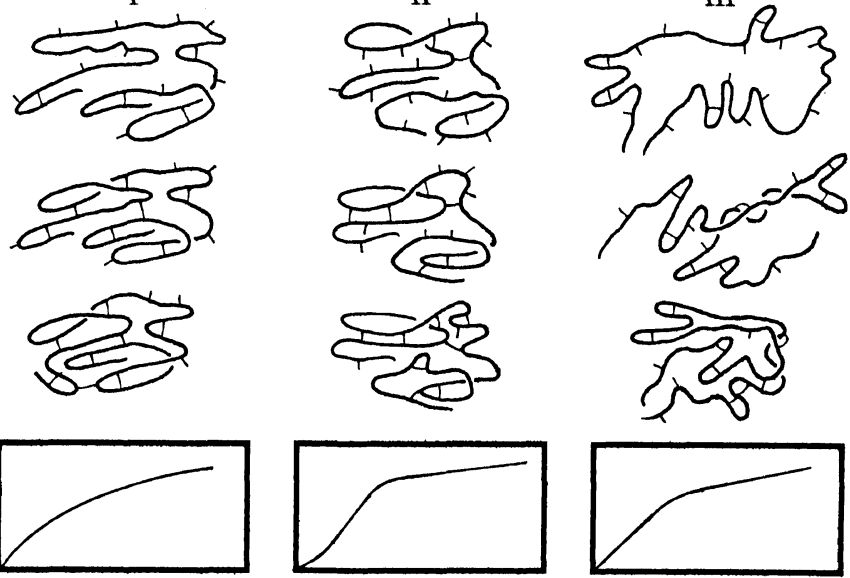

Irradiation time
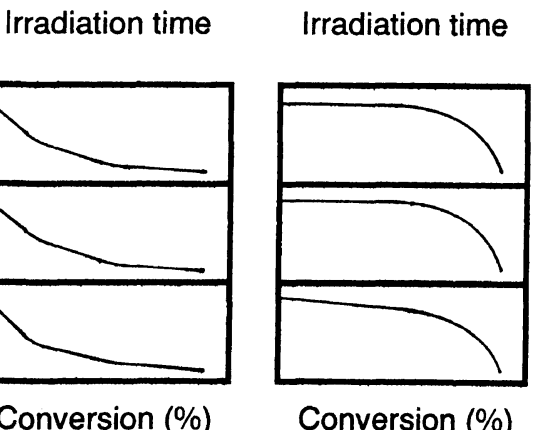

Figure 11. Changes in the structure of polymer coil and $\left\langle S^{2}\right\rangle^{1 / 2}, D_{\mathrm{r}}$, and $D_{\mathrm{s}}$ corresponding to three possible models for an intramacromolecular crosslinking reaction. I, random reaction model; II, long range reaction model; III, short range reaction model. 
crosslinking reaction, and discuss which is the most suitable to describe the process of the present intramacromolecular photo-crosslinking reaction. Changes in values of $\left\langle S^{2}\right\rangle^{1 / 2}$, $D_{\mathrm{r}}$, and $D_{\mathrm{s}}$ against conversion of anthryl moiety are expected for each model as shown in Figure 11 together with the schematic illustration of the changes in the polymer coils.

Random Reaction Model. For this model, the reaction is described as the sum of some reactions with randomly distributed rate constants. Of course the reaction site with large rate constant reacts fast and that with small rate constant reacts slowly but these sites are distributed randomly. So the reaction cannot be described by two stages of reaction. The mobilities of the polymer chains and dimension of the polymer coil are expected to decrease proportionally to the number of crosslinked side chains.

Long Range Reaction Model. In the initial stage of the reaction, crosslinks may be formed at points where crosslinkable side chains exist spatially near-by each other in an individual polymer coil but far-away along the chain contour. At this stage a large shrinkage of the polymer coil may occur and mobility of the polymer chain is suppressed greatly. When some crosslinks are formed, the next crosslinking reaction may proceed faster, because the polymer coil shrinks and the probability of collision in the polymer coil increases. In the course of the reaction there is a stage where the reaction is accelerated. Because the crosslinking reaction proceeds in the shrank polymer coil, in this stage the radius of gyration of the polymer coil decreases and mobility of the polymer chain is suppressed more slowly than the initial stage. In time, the rate of crosslinking reaction will become slower, because the mobility of crosslinkable side chains is suppressed and they don't contact each other. The mobility of polymer chains and dimensions of polymer coil may change slightly.

Short Range Reaction Model. This model consists of two stages like a folding process for proteins. In the initial stage, crosslinks may be formed between nearest-neighbor crosslinkable side chains, where fast crosslinking reactions take place. Hence translational motion of the chain segments is gradually suppressed. In time, crosslinks between far-away side chains are formed since there remain no crosslinkable side chain to form the nearestneighbor crosslinks. Therefore, the polymer shrinks only in the later stage. The mobility of polymer chain is suppressed mainly in the later stage.

Changes in structure and parameters for polymer chain dynamics of the polymer shown in Figure 10 is very similar to the change in the third "short range reaction model" in Figure 11. The structural change in the polymer proceeds along the short range reaction process.

The short range reaction model might have some analogy to the folding process of protein. Generally the protein folding process cannot be described by thermodynamics. The denaturated ribonuclease $\mathrm{A}$ is known to refold through a series of processes with different rate constants. ${ }^{17-20}$ There is the possibility that a synthetic polymer proceeds its intramolecular reaction along the process similar to the protein folding. The short range reaction process in the system is anticipated to correspond to so-called framework process for protein folding, ${ }^{21,22}$ which consists of the following steps; 1) secondary structures such as $\alpha$-helix and $\beta$-sheet are formed rapidly by the short-range interactions, 2) the secondary structures are packed into a molten globule state, where the structure of the protein fluctuates while it maintains the secondary structures, 3) the distance between hydrophobic residues becomes shorter, then the molten globule intermediate transforms slowly into a specific tertiary structure due to the hydrophobic interaction. ${ }^{23}$

In conclusion, the intramacromolecular photo-crosslinking reaction in the experimen- 
tal system may proceed along the process below. In the initial stage crosslinks are formed between the nearest-neighbor side chains along the polymer chain contour. In this stage, the dimensions of the polymer coil change little and translational motion is suppressed slightly. In time, reactive side chains located nearest-neighbor along the chain are consumed by the reaction. The crosslinks are formed between far-apart side chains still remaining from the reaction. The polymer coils shrink and the density of monomer units increases inside the polymer coils, hence rotational and translational motion are suppressed. The preparation of the photocrosslinkable polymers with emission probe and evaluation of various dynamic parameters during the photo-crosslinking reaction make it possible to clarify the polymer folding process in a synthetic polymer system.

\section{REFERENCES}

1. A. Reiser and P. L. Egerton, Photogr. Sci. Enging., 23, 144 (1979).

2. A. Reiser and P. L. Egerton, Macromolecules, 12 670 (1979).

3. P. L. Egerton, E. Pitts, and A. Reiser, Macromolecules, 14, 95 (1981).

4. E. Pitts and A. Reiser, J. Am. Chem. Soc., 105, 5590 (1983).

5. Y. Shindo, T. Sugimura, and K. Horie, Eur. Polym.
J., 25, 1033 (1989).

6. Y. Shindo, T. Sugimura, K. Horie, and I. Mita, Polym. J., 22, 63 (1990).

7. Y. Shindo, T. Sugimura, K. Horie, and I. Mita, Eur. Polym. J., 26, 221 (1990).

8. M. A. Winnik, Ed., "Photophysical and Photochemical Tools in Polymer Science," NATO ASI Series C, 1986, p 182.

9. S. Kondo, T. Ohtsuka, K. Ogura, and K. Tsukada, J. Macromol. Sci.-Chem., A13, 767 (1979).

10. J. Pacrkowski and D. C. Neckers, Macromolecules, 18, 1245 (1985).

11. Z. Grubisic, P. Rempp, and H. Benoit, J. Polym. Sci., B5, 753 (1967).

12. P. J. Flory and T. G. Fox, J. Am. Chem. Soc., 73, 1904 (1951).

13. G. Weber, Adv. Protein Chem., 8, 415 (1953).

14. H. Ushiki and I. Mita, Polym. J., 16, 751 (1984).

15. J. S. Hargreaves and S. E. Webber, Macromolecules, 17, 235 (1984).

16. I. Mita and K. Horie, J. Macromol. Sci.-Rev. Macromol. Chem. Phys., C27, 91 (1987).

17. J. R. Garel and R. L. Baldwin, Proc. Natl. Acad. Sci. U.S.A., 70, 3347 (1973).

18. J. R. Garel and R. L. Baldwin, J. Mol. Biol., 94, 611 (1975).

19. L.-N. Lin and J. F. Brandts, Biochemistry, 22, 564 (1983).

20. P. W. Mui, Y. Konishi, and H. A. Sheraga, Biochemistry, 24, 4481 (1985).

21. O. B. Ptitsyn and A. A. Rashin, Biophys. Chem., 3, 9 (1975).

22. P. S. Kim and R. L. Baldwin, Ann. Rev. Biochem., 51, 459 (1982).

23. N. Saito, T. Shigaki, Y. Kobayashi, and $\mathbf{M}$. Yamamoto, Proteins: Struct. Funct. Gen., 3, 199 (1988). 